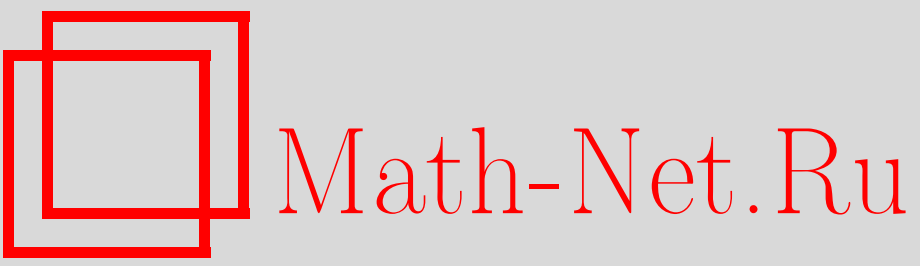

О. А. Чалых, Преобразования Дарбу для многомерных операторов Шрёдингера, УМH, 1998, том 53, выпуск 2, 167-168

DOI: https://doi.org/10.4213/rm33

Использование Общероссийского математического портала Math-Net.Ru подразумевает, что вы прочитали и согласны с пользовательским соглашением

http://www.mathnet.ru/rus/agreement

Параметры загрузки:

IP : 54.205 .225 .156

26 апреля 2023 г., 13:25:28 


\title{
ПРЕОБРАЗОВАНИЯ ДАРБУ ДЛЯ МНОГОМЕРНЫХ ОПЕРАТОРОВ ШРЁДИНГЕРА
}

\author{
О. А. ЧАлых
}

Рассмотрим конечный набор $A$ различных гиперплоскостей в $\mathbb{C}^{n}$, каждая из которых определяется уравнением вида

$$
\alpha(x)=a_{0}+a_{1} x_{1}+\cdots+a_{n} x_{n}=0, \quad a_{i} \in \mathbb{C},
$$

и предполагается неизотропной, т.е. нормаль $\alpha^{\perp}=\left(a_{1}, \ldots, a_{n}\right)$ имеет ненулевой квадрат: $\left(\alpha^{\perp}, \alpha^{\perp}\right)=a_{1}^{2}+\cdots+a_{n}^{2} \neq 0$. Пусть каждой гиперплоскости $\alpha \in A$ приписана кратность $m_{\alpha} \in \mathbb{Z}_{+}$. Такой конфигурации гиперплоскостей сопоставим следующий оператор Шредингера в $\mathbb{C}^{n}$ :

где

$$
L_{A}=\Delta-u(x),
$$

$$
u(x)=\sum_{\alpha \in A} \frac{m_{\alpha}\left(m_{\alpha}+1\right)\left(\alpha^{\perp}, \alpha^{\perp}\right)}{(\alpha(x))^{2}}, \quad \Delta=\frac{\partial^{2}}{\partial x_{1}^{2}}+\cdots+\frac{\partial^{2}}{\partial x_{n}^{2}} .
$$

Мы хотим описать те из операторов $L_{A}$, которые связаны с оператором Лапласа $L_{0}=\Delta$ некоторым преобразованием Дарбу. Под этим мы понимаем наличие дифференциального оператора $D(x, \partial / \partial x)$ такого, что

$$
L_{A} \circ D=D \circ L_{0}
$$

В размерности $n=1$ это эквивалентно тому, что оператор $L_{A}$ получается из $L_{0}$ конечным числом классических преобразований Дарбу (см., например, [1]). Отметим, что существование сплетающего оператора $D$ эквивалентно наличию у $L_{A}$ собственной функции $\psi$ вида $\psi(k, x)=$ $P(k, x) \exp \left(k_{1} x_{1}+\cdots+k_{n} x_{n}\right)$, где $P(k, x)$ - полином по $k=\left(k_{1}, \ldots, k_{n}\right), L_{A} \psi=k^{2} \psi$ : чтобы получить $\psi$, надо подействовать оператором $D$ на $\exp (k, x)$.

Для того, чтобы сформулировать соответствующие условия на конфигурацию, разложим потенциал $u(x)$ в ряд Лорана по нормали к гиперплоскости $\alpha \in A$, т.е. по степеням $z=\alpha(x)$ : $u=m_{\alpha}\left(m_{\alpha}+1\right)\left(\alpha^{\perp}, \alpha^{\perp}\right) z^{-2}+\sum_{i \geqslant 0} c_{i} z^{i}$, где $c_{i}$ - функции на гиперплоскости $\alpha(x)=0$. Можно показать, что также как и в одномерном случае [1], необходимым условием существования сплетающего оператора $D$ является обнуление коэффициентов $c_{1}, c_{3}, \ldots, c_{2} m_{\alpha}-1$ на соответствующей гиперплоскости (см. [2]). Оказывается, это условие является и достаточнылм.

Теорема 1. Если в лорановском разложении потенциала и по нормали к любой из гиперплоскостей $\alpha \in A$ отсутствуют нечетнье степени $z^{2 j-1}$ с $j=1,2, \ldots, m_{\alpha}$, то оператор $L_{A}$ связан с оператором Лапласа некоторым преобразованием Дарбу.

Условия теоремы эквивалентны следующим алгебраическим соотношениям для набора $\left\{\alpha, m_{\alpha}\right\}:$ на любой гиперплоскости $\alpha(x)=0$ следующее выражение есть тождественный ноль:

$$
\sum_{\beta \in A \backslash \alpha} m_{\beta}\left(m_{\beta}+1\right)\left(\beta^{\perp}, \beta^{\perp}\right)\left(\alpha^{\perp}, \beta^{\perp}\right)^{2 j-1}(\beta(x))^{-2 j-1} \equiv 0 \text { при } j=1, \ldots, m_{\alpha} .
$$

(Сp. с [1], где эти соотношения были получены в случае $n=1$.)

Для доказательства теоремы рассмотрим следующее линейное пространство $V$, которое состоит из всевозможных функций $f(k, x)$ со следующими аналитическими свойствами: 1$) f$ имеет вид $Q(k, x) \prod_{\alpha \in A}(\alpha(x))^{-m_{\alpha}} \exp (k, x)$, где $Q$ - многочлен по $k$ и $\left.x ; 2\right)$ в лорановском разложении $f$ как функции от $x$ по нормали к любой из гиперплоскостей $\alpha \in A$ отсутствуют члены вида $z^{2 j-1-m_{\alpha}}$ с $j=1, \ldots, m_{\alpha}$.

Следующее наблюдение является ключевым и немедленно вытекает из свойств лорановских разложений для $u(x)$ и $f \in V$ :

Работа выполнена при поддержке Российского фонда фундаментальных исследований (гранты № № 96-01-01404 и 96-15-96027) и INTAS (грант № № 93-166-ext и N96-0770). 
Лемма. В предположениях теоремы 1 пространство $V$ инв ариантно относительно действия оператора $L_{A}$.

Рассмотрим теперь функцию $f_{0}=\prod_{\alpha \in A}(\alpha(x))^{m_{\alpha}} \exp (k, x)$, очевидно, лежащую в $V$. Согласно лемме, для любого $i>0$ функция $f_{i}=\left(L_{A}-k^{2}\right)^{i} f_{0}$ также лежит в $V$. При этом $f_{i}$ имеет вид $Q_{i} \prod_{\alpha \in A}(\alpha(x))^{-m_{\alpha}} \exp (k, x)$, где степени по $x$ многочленов $Q_{i}$ монотонно убьвают с возрастанием $i$. Значит, для некоторого $M f_{M} \neq 0$, а $f_{M+1}=\left(L_{A}-k^{2}\right) f_{M}=0$. Поэтому $\psi=f_{M}$ является собственной для оператора $L_{A}$, что и доказывает теорему.

Нетрудно показать, что на самом деле $M=\sum_{\alpha \in A} m_{\alpha}$, тем самым $\psi$-функция задается следующей явной формулой: $\psi=\left(L_{A}-k^{2}\right)^{M}\left[\prod_{\alpha \in A}(\alpha(x))^{m_{\alpha}} \exp (k, x)\right]$. Эта замечательная формула возникла впервые (в предположениях существования $\psi$ ) у Ю. Ю. Береста [3], и именно она подсказала основную идею данной работы.

Справедлив также следующий тригонометрический аналог теоремы 1 , когда данному (конечному) набору гиперплоскостей в $\mathbb{C}^{n}$ сопоставляется оператор $L_{A}^{\omega}=\Delta-u(x)$, где $u(x)=$ $\sum_{\alpha \in A} \frac{m_{\alpha}\left(m_{\alpha}+1\right) \omega^{2}\left(\alpha^{\perp}, \alpha^{\perp}\right)}{\sin ^{2} \omega \alpha(x)}, \omega \neq 0$ - произволшный параметр. Мы предполагаем, что $u(x)$ имеет $n$ (линейно независимых над $\mathbb{C}$ ) периодов $t_{1}, \ldots, t_{n},\left(\omega \alpha^{\perp}, t_{i}\right) \in \pi \mathbb{Z}$ для всех $\alpha$, а также, что при $\alpha \neq \beta$ и $l, m \in \mathbb{Z}$ гиперплоскости $\alpha(x)=l \pi / \omega$ и $\beta(x)=m \pi / \omega$ различны.

ТеОРема 2. Если для тригонометрического потенциала и $(x)$ условия теоремь 1 выполняются на любой из гиперплоскостей $\alpha(x)=l \pi / \omega$ c $l \in \mathbb{Z}$, то соответствующий оператор $L_{A}^{\omega}$ связан с оператором Лапласа некоторым преобразованием Дарбу $D$ c периодическими коэффициентами.

Доказательство основывается на той же идее, но выражение для $\psi$-функции имеет бо лее сложный вид. В случае $n=1$ утверждение теоремы ранее было получено другим способом [4] на основании результатов [5].

ЗАмЕчАния. 1) Естественные примеры конфигураций, удовлетворяющих условиям теорем 1 и 2 , доставляют зеркала групп Кокстера и, соответственно, системы корней с инвариантными кратностями $m_{\alpha}$. В этом случае известен другой способ получения $\psi$-функции с помощью так называемых операторов сдвига [6], [7]. Наш подход дает новьй способ построения таких операторов. Другие примеры конфигураций см. в работах [5], [8].

2) Используя результаты [2] и [9], можно показать, что теорема 1 описывает все операторы Шрёдингера с рациональным потенциалом, которые связаны с оператором Лапласа некоторым преобразованием Дарбу.

Я глубоко признателен Ю. Ю. Бересту, А. П. Веселову и М. В. Фейгину за чрезвычайно плодотворные обсуждения.

\section{СПИСОК ЛИТЕРАТУРЫ}

[1] Duistermaat J. J., Grünbaum F. A. // Comm. Math. Phys. 1986. V. 103. P. 177-240. [2] Фейгин М. В. Дипломная работа. М.: МГУ, 1997. [3] Berest Yu. // CRM Proceedings and Lecture Notes 1998. V. 14. Р. 9-28. [4] Берест Ю. Ю., Веселов А. П. Частное сообщение. [5] Berest Yu. // Comm. Pure Appl. Math. 1997. V. 50. № 10. P. 1021-1054. [6] Opdam E. M. // Compositio Math. 1988. V. 67. № 2. P. 191-209. [7] Heckman G. J. // Progr. Math. 1991. V. 101. Р. 181-191. [8] Веселов А. П., Фейгин М. В., Чалых О.А. // УМН. 1996. Т. 51. № 3. С. 185-186. [9] Берест Ю. Ю., Веселов А. П. // УМН. 1998. Т. 53. № 1. С. 211-212. 